\title{
On the Minimum Variable Connectivity Index of Unicyclic Graphs with a Given Order
}

\author{
Shamaila Yousaf, ${ }^{1,2}$ Akhlaq Ahmad Bhatti $\mathbb{D}^{1},{ }^{1}$ and Akbar Ali $\mathbb{D}^{3,4}$ \\ ${ }^{1}$ Department of Sciences and Humanities, National University of Computer and Emerging Sciences, Lahore Campus, B-Block, \\ Faisal Town, Lahore, Pakistan \\ ${ }^{2}$ Department of Mathematics, University of Gujrat, Hafiz Hayat Campus, Gujrat, Pakistan \\ ${ }^{3}$ College of Science, University of Hail, Hail 81451, Saudi Arabia \\ ${ }^{4}$ Knowledge Unit of Science, University of Management and Technology, Sialkot, Pakistan
}

Correspondence should be addressed to Akbar Ali; akbarali.maths@gmail.com

Received 13 April 2020; Accepted 19 June 2020; Published 18 July 2020

Academic Editor: Miguel Ángel López

Copyright ( $) 2020$ Shamaila Yousaf et al. This is an open access article distributed under the Creative Commons Attribution License, which permits unrestricted use, distribution, and reproduction in any medium, provided the original work is properly cited.

The variable connectivity index, introduced by the chemist Milan Randić in the first quarter of 1990s, for a graph $G$ is defined as $\sum_{v w \in E(G)}\left(\left(d_{v}+\gamma\right)\left(d_{w}+\gamma\right)\right)^{-1 / 2}$, where $\gamma$ is a non-negative real number and $d_{w}$ is the degree of a vertex $w$ in $G$. We call this index as the variable Randić index and denote it by ${ }^{v} R_{\gamma}$. In this paper, we show that the graph created from the star graph of order $n$ by adding an edge has the minimum ${ }^{v} R_{\gamma}$ value among all unicyclic graphs of a fixed order $n$, for every $n \geq 4$ and $\gamma \geq 0$.

\section{Introduction}

All graphs that we discuss in the present study are simple, connected, undirected, and finite. For a graph $G=(V, E)$, the number $|V(G)|$ is called its order and $|E(G)|$ is called its size. For a vertex $v_{1} \in V(G)$, denoted by $N\left(v_{1}\right)$, the set of all those vertices of $G$ are adjacent with $v_{1}$. The number $d_{v_{1}}=$ $\left|N\left(v_{1}\right)\right|$ is called degree of $v_{1}$. If $d_{v_{1}}=1$, then $v_{1}$ is called a pendent vertex or a leaf. A graph of order $n$ is known as an $n$-vertex graph. As usual, the $n$-vertex path and star graphs are denoted by $P_{n}$ and $S_{n}$, respectively. An $n$-vertex graph containing exactly one cycle is called a unicyclic graph. The class of all $n$-vertex unicyclic graphs is denoted by $U_{n}$. The graph obtained from $S_{n}$ by adding an edge is denoted by $S_{n}^{1}$. For the (chemical) graph theoretical notation and terminology that are not defined in this paper, we refer the reader to some standard books, such as [1-3].

To model the heteroatoms molecules, it is better to use the vertex-weighted graphs, which are the graphs whose one or more vertices are distinguished in some way from the rest of the vertices [4]. Let $G$ be a vertex-weighted graph with the vertex set $\left\{v_{1}, v_{2}, \ldots, v_{n}\right\}$ and the vertex weight $w_{i}$ of the vertex $v_{i}$ is for $i=1,2, \ldots, n$. The augmented vertex-adjacency matrix of $G$ is an $n \times n$ matrix denoted by ${ }^{a v} \mathbf{A}(G)$ and is defined as ${ }^{a v} \mathbf{A}(G)=\left[a_{i, j}\right]_{n \times n}$, where

$$
a_{i, j}= \begin{cases}1, & \text { if } v_{i} v_{j} \in E(G), \\ w_{i}, & \text { if } i=j \\ 0, & \text { otherwise }\end{cases}
$$

The variable connectivity index $[5,6]$, proposed by Randić, for the graph $G$ is defined as

$$
{ }^{1} \chi^{f}(G)=\sum_{v_{i} v_{j} \in E(G)} \frac{1}{\sqrt{\left(d_{v_{i}}+w_{i}\right)\left(d_{v_{j}}+w_{j}\right)}} .
$$

We associate this index's name with its inventor Randić by calling it as the variable Randić index. This index was actually introduced within the QSPR/QSAR (quantitative structure-property/activity relationship) studies of heteroatoms molecules. If $G$ is the molecular graph of a homoatomic molecule, then $w_{1}=w_{2}=\cdots=w_{n}=\gamma$ (say) and hence the variable Randić index ${ }^{1} \chi^{f}(G)$ becomes 


$$
{ }^{1} \chi^{f}(G)=\sum_{v_{i} v_{j} \in E(G)} \frac{1}{\sqrt{\left(d_{v_{i}}+\gamma\right)\left(d_{v_{j}}+\gamma\right)}}
$$

In the rest of this paper, we denote this index by ${ }^{v} R_{\gamma}$ instead of ${ }^{1} \chi^{f}$. Clearly, if we take $\gamma=0$ then the invariant ${ }^{v} R_{\gamma}(G)$ is the classical Randić index [7, 8]. Details about the chemical applications of the variable Randić index can be found in $[4,7,9-17]$ and related references listed therein. In [18], a mathematical study of the variable Randić index was initiated and it was proved that the star graph has the minimum variable Randić index among all trees of a fixed order $n$, where $n \geq 4$. It needs to be mentioned here that the variable Randić index seems to have more chemical applications than the several wellknown variable indices, see, for example, the variable indices considered in the papers [19-25].

For convenience, we introduce some further notation and terminology. Let $A_{n, l, j}^{k}$ be the unicyclic graph constructed from a path $P=u_{0} u_{1} \ldots u_{j}(j \geq 1)$ by connecting $k$ pendant vertices to $u_{0}$ and a cycle $C_{l}, l=n-j-k$ to $u_{j}$, respectively. Let $A_{n, l}^{k}$ be the unicyclic graph constructed from cycle $C_{l}, l=n-k$ by connecting $k$ pendant vertices to one vertex of $C_{l}$ and $A_{n, l}^{i, j, k}$ be the unicyclic graph created from cycle $C_{l}, n=i+j+k+l$ by attaching $K_{1, i}, K_{1, j}$, and $K_{1, k}$ to the vertices of $C_{l}$. Let $\mathbf{D}$ be the such class of $U_{n}$ whose every member has unique 3-cycle, and the vertices apart from 3-cycle are pendent vertices. Let $\mathbf{F}$ be the such class of $U_{n}$ whose every member has unique 4-cycle, and the vertices apart from 4-cycle are pendent vertices and are joined to nonadjacent vertices of the unique 4-cycle.

In this paper, we characterize the collection of unicyclic graphs on $n$ vertices that minimize variable Randić index. We further show that, for $\gamma \geq 0, S_{n}^{1}$ has minimum variable Randić index among the collection $U_{n}$ and

$$
\begin{aligned}
{ }^{v} R_{\gamma}(G) \geq & \frac{n-3}{\sqrt{(1+\gamma)(n-1+\gamma)}}+\frac{2}{\sqrt{(2+\gamma)(n-1+\gamma)}} \\
& +\frac{1}{\sqrt{(2+\gamma)(2+\gamma)}},
\end{aligned}
$$

where equality holds if and only if $G \cong S_{n}^{1}$.

\section{Transformations Which Decrease the Variable Randić Index}

We introduce some transformations and prove some lemmas to establish main results.

Lemma 1. If $\gamma \geq 0$ and $s, t \geq 1$, then the function $\Psi$ defined as

$$
\begin{aligned}
\Psi(\gamma, s, t)= & \frac{s}{\sqrt{1+\gamma}}\left(\frac{1}{\sqrt{s+2+\gamma}}-\frac{1}{\sqrt{s+t+3+\gamma}}\right) \\
& +\frac{t}{\sqrt{1+\gamma}}\left(\frac{1}{\sqrt{t+2+\gamma}}-\frac{1}{\sqrt{s+t+3+\gamma}}\right) \\
& +\frac{1}{\sqrt{(s+2+\gamma)(t+2+\gamma)}} \\
& -\frac{1}{\sqrt{(1+\gamma)(s+t+3+\gamma)}}
\end{aligned}
$$

is positive valued.

Proof. We note that the function $\partial \Psi / \partial s$ is strictly increasing in $t$ on the interval $(1, \infty)$ because

$$
\begin{aligned}
\frac{\partial \Psi}{\partial s}= & \frac{1}{\sqrt{\gamma+1}}\left(\frac{1}{\sqrt{s+2+\gamma}}-\frac{1}{\sqrt{s+t+3+\gamma}}\right) \\
& +\frac{s}{2 \sqrt{\gamma+1}}\left(\frac{1}{(s+t+3+\gamma)^{3 / 2}}-\frac{1}{(s+2+\gamma)^{3 / 2}}\right) \\
& +\frac{t}{2 \sqrt{\gamma+1}(s+t+3+\gamma)^{3 / 2}} \\
& -\frac{1}{2 \sqrt{t+2+\gamma}(s+2+\gamma)^{3 / 2}} \\
& +\frac{1}{2 \sqrt{\gamma+1}(s+t+3+\gamma)^{3 / 2}}, \\
\frac{\partial t}{\partial s}\left(\frac{\partial \Psi}{\partial s}\right)= & \frac{1}{4}\left(\frac{1}{(s+2+\gamma)^{3 / 2}(t+2+\gamma)^{3 / 2}}\right. \\
& \left.+\frac{s+t+9+4 \gamma}{\sqrt{1+\gamma}(s+t+3+\gamma)^{5 / 2}}\right)>0 .
\end{aligned}
$$

Also, it can be seen that the value of the function $\partial \Psi / \partial s$ at $t=1$ is $>0$, which implies that the function $\partial \Psi / \partial s$ is positive valued for $t>1$, and hence the function $\Psi$ is strictly increasing w.r.t. $s$ on the interval $(1, \infty)$. In our case $\Psi(\gamma, s, t)=\Psi(\gamma, s, t)$, the function $\Psi$ is also strictly increasing w.r.t. $t$ on the interval $(1, \infty)$. Hence, $\Psi(\gamma, s, t)>0$ for all $s, t>1$ and $\gamma \geq 0$.

Lemma 2. If $\gamma \geq 0$ and $s, t \geq 1$, then the function $\Phi$ defined as

$$
\begin{aligned}
\Phi(\gamma, s, t)= & \frac{s}{\sqrt{1+\gamma}}\left(\frac{1}{\sqrt{s+2+\gamma}}-\frac{1}{\sqrt{s+t+4+\gamma}}\right) \\
& +\frac{t}{\sqrt{1+\gamma}}\left(\frac{1}{\sqrt{t+2+\gamma}}-\frac{1}{\sqrt{s+t+4+\gamma}}\right) \\
& +\frac{1}{\sqrt{(s+2+\gamma)(2+\gamma)}}-\frac{1}{\sqrt{(1+\gamma)(s+t+4+\gamma)}} \\
& +\frac{1}{\sqrt{(t+2+\gamma)(2+\gamma)}}-\frac{1}{\sqrt{(1+\gamma)(s+t+4+\gamma)}},
\end{aligned}
$$

is positive valued.

The proof of Lemma 2 is analogous to the proof of Lemma 1.

Lemma 3. If $\gamma \geq 0, t>2$, and $r>1$, it holds that 


$$
\begin{aligned}
\Omega(\gamma, r, t)= & \frac{r-1}{\sqrt{1+\gamma}}\left(\frac{1}{r+\gamma}-\frac{1}{\sqrt{r+t+\gamma}}\right) \\
& +\frac{1}{\sqrt{(r+\gamma)(t+1+\gamma)}}-\frac{1}{\sqrt{(1+\gamma)(r+t+\gamma)}}>0 .
\end{aligned}
$$

$$
\begin{aligned}
\Omega(\gamma, r, t)= & \frac{(r-1)(t-1)}{\sqrt{(1+\gamma)(r+\gamma)(t+\gamma)(r+t+\gamma)}} \\
& \times\left(\frac{\sqrt{t+\gamma}}{\sqrt{r+\gamma}+\sqrt{r+t-1+\gamma}}-\frac{1}{\sqrt{(r+\gamma)(t+\gamma)}+\sqrt{(1+\gamma)(r+t-1+\gamma)}}\right) \\
= & \left(\frac{(r-1)(t-1)}{\sqrt{(1+\gamma)(r+\gamma)(t+\gamma)(r+t-1+\gamma)}}\right) \\
& \times\left[\frac{\sqrt{t+\gamma}(\sqrt{(r+\gamma)(t+\gamma)}+\sqrt{(1+\gamma)(r+t-1+\gamma)})}{(\sqrt{r+\gamma}+\sqrt{r+t-1+\gamma})(\sqrt{(r+\gamma)(t+\gamma)}+\sqrt{(1+\gamma)(r+t-1+\gamma)})}\right) \\
& \left.-\left(\frac{(\sqrt{r+\gamma}+\sqrt{r+t-1+\gamma})}{(\sqrt{r+\gamma}+\sqrt{r+t-1+\gamma})(\sqrt{(r+\gamma)(t+\gamma)}+\sqrt{(1+\gamma)(r+t-1+\gamma)})}\right)\right] \\
& \times\left(\frac{(r-1)(t-1)}{(\sqrt{r+\gamma}+\sqrt{r+t-1+\gamma})(\sqrt{(r+\gamma)(t+\gamma)}+\sqrt{(1+\gamma)(r+t-1+\gamma)})}\right) .
\end{aligned}
$$

Since $\gamma \geq 0, t>2$ so $t+\gamma>0$, also $(\sqrt{1+\gamma} \sqrt{t+\gamma}-1)>0$. Hence, $\Omega(\gamma, r, t)>0$.

Lemma 4. For $\gamma \geq 0$, it holds that

$$
\begin{aligned}
f(\gamma)= & \frac{2}{\sqrt{2+\gamma}}\left(\frac{1}{\sqrt{\gamma+2}}-\frac{1}{\sqrt{\gamma+3}}\right) \\
& +\frac{1}{\sqrt{(2+\gamma)(2+\gamma)}}-\frac{1}{\sqrt{(1+\gamma)(\gamma+3)}}>0 .
\end{aligned}
$$

The proof of Lemma 4 is analogous to the proof of Lemma 3.

Lemma 5. For $\gamma \geq 0$, it holds that

$$
\begin{aligned}
f(\gamma)= & \frac{1}{\sqrt{1+\gamma}}\left(\frac{1}{\sqrt{\gamma+3}}-\frac{1}{\sqrt{\gamma+4}}\right)+\frac{2}{\sqrt{2+\gamma}}\left(\frac{1}{\sqrt{\gamma+3}}-\frac{1}{\sqrt{\gamma+4}}\right) \\
& +\frac{1}{\sqrt{(2+\gamma)(2+\gamma)}}-\frac{1}{\sqrt{(1+\gamma)(\gamma+4)}}>0 .
\end{aligned}
$$

The proof of Lemma 5 is analogous to the proof of Lemma 3.

Now, we use three transformations, which will reduce the variable Randić index.
2.1. Transformation 1. Let $G \in U_{n}$ and $u v$ be an edge of $G$ such that $d_{v}=r \geq 2 . N(v)=\left\{u, w_{1}, w_{2}, \ldots, w_{r-1}\right\}$, where $w_{1}, w_{2}, \ldots, w_{r-1}$ are leaves.

Define $\stackrel{G}{=}=G-\left\{v w_{1}, v w_{2}, \ldots, v w_{r-1}\right\}+\left\{u w_{1}, u w_{2}, \ldots\right.$, $\left.u w_{r-1}\right\} . G$ and $G$ are depicted in Figure 1. 


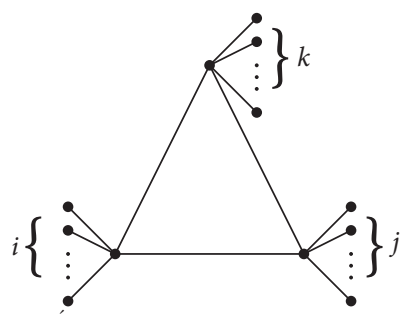

Figure 1: The graph $G$ (obtained using Transformation 1) from $G$.

Lemma 6. Let $G, G \in U_{n}$. By applying Transformation 1 and Proof. Let $d_{u}=t \geq 3$ : for $\gamma \geq 0$, we have

$$
{ }^{v} R_{\gamma}(G)>{ }^{v} R_{\gamma}(\dot{G})
$$

$$
\begin{aligned}
& { }^{v} R_{\gamma}(G)-{ }^{v} R_{\gamma}(\dot{G})>\frac{d_{v}-1}{\sqrt{(1+\gamma)\left(d_{v}+\gamma\right)}}+\frac{1}{\sqrt{\left(d_{v}+\gamma\right)\left(d_{u}+\gamma\right)}}-\frac{d_{v}}{\sqrt{(1+\gamma)\left(d_{v}+d_{u}-1+\gamma\right)}}, \\
& { }^{v} R_{\gamma}(G)-{ }^{v} R_{\gamma}(\dot{G})>\frac{d_{v}-1}{\sqrt{1+\gamma}}\left(\frac{1}{\sqrt{d_{v}+\gamma}}-\frac{1}{\sqrt{d_{v}+d_{u}-1+\gamma}}\right)+\frac{1}{\sqrt{\left(d_{v}+\gamma\right)\left(d_{u}+\gamma\right)}}-\frac{1}{\sqrt{(1+\gamma)\left(d_{v}+d_{u}-1+\gamma\right)}}, \\
& { }^{v} R_{\gamma}(G)-{ }^{v} R_{\gamma}(\dot{G})>\frac{r-1}{\sqrt{1+\gamma}}\left(\frac{1}{\sqrt{r+\gamma}}-\frac{1}{\sqrt{t+r-1+\gamma}}\right)+\frac{1}{\sqrt{(r+\gamma)(t+\gamma)}}-\frac{1}{\sqrt{(1+\gamma)(t+r-1+\gamma)}}>0 .
\end{aligned}
$$

Using Lemma 3, one can see that inequality (13) holds.

2.2. Transformation 2. Let $G, G \in U_{n}$ such that $|G|=|\dot{G}|$ and their unique cycles are $r$-cycle and $(r-1)$-cycle, respectively, $r \geq 4$. For both graphs, the vertices apart from the cycle are leaf vertices joined to exactly one vertex of the cycle.
Lemma 7. Let $G, \dot{G} \in U_{n}$ such that $|G|=|\dot{G}|$. By applying Transformation 2 and for $\gamma \geq 0$, we have

$$
{ }^{v} R_{\gamma}(G)>{ }^{v} R_{\gamma}(\dot{G}) \text {. }
$$

Proof. Since

$$
\begin{aligned}
{ }^{v} R_{\gamma}(G)= & \frac{r-2}{\sqrt{(\gamma+2)(\gamma+2)}}+\frac{n-r}{\sqrt{(\gamma+1)(n-r+\gamma+2)}}+\frac{2}{\sqrt{(\gamma+2)(n-r+\gamma+2)}}, \\
{ }^{v} R_{\gamma}(\dot{G})= & \frac{r-3}{\sqrt{(\gamma+2)(\gamma+2)}}+\frac{n-r+1}{\sqrt{(\gamma+1)(n-r+\gamma+3)}}+\frac{2}{\sqrt{(\gamma+2)(n-r+\gamma+3)}}, \\
{ }^{v} R_{\gamma}(G)-{ }^{v} R_{\gamma}(\dot{G})= & \frac{n-r}{\sqrt{1+\gamma}}\left(\frac{1}{\sqrt{n-r+\gamma+2}}-\frac{1}{\sqrt{n-r+\gamma+3}}\right) \\
& +\frac{2}{\sqrt{2+\gamma}}\left(\frac{1}{\sqrt{n-r+\gamma+2}}-\frac{1}{\sqrt{n-r+\gamma+3}}\right) \\
& +\frac{1}{\sqrt{(2+\gamma)(2+\gamma)}}-\frac{1}{\sqrt{(1+\gamma)(n-r+\gamma+3)}} .
\end{aligned}
$$


Now, there will be three cases.

Case I: if $n=r$, then

$$
\begin{aligned}
{ }^{v} R_{\gamma}(G)-{ }^{v} R_{\gamma}(\dot{G})= & \frac{2}{\sqrt{2+\gamma}}\left(\frac{1}{\sqrt{\gamma+2}}-\frac{1}{\sqrt{\gamma+3}}\right) \\
& +\frac{1}{\sqrt{(2+\gamma)(2+\gamma)}}-\frac{1}{\sqrt{(1+\gamma)(\gamma+3)}}
\end{aligned}
$$

Using Lemma 4, one can see that relation (16) is positive valued.

Case II: if $n-r=1$, then

$$
\begin{aligned}
{ }^{v} R_{\gamma}(G)-{ }^{v} R_{\gamma}(G)= & \frac{1}{\sqrt{1+\gamma}}\left(\frac{1}{\sqrt{\gamma+3}}-\frac{1}{\sqrt{\gamma+4}}\right) \\
& +\frac{2}{\sqrt{2+\gamma}}\left(\frac{1}{\sqrt{\gamma+3}}-\frac{1}{\sqrt{\gamma+4}}\right) \\
& +\frac{1}{\sqrt{(2+\gamma)(2+\gamma)}}-\frac{1}{\sqrt{(1+\gamma)(\gamma+4)}}
\end{aligned}
$$

Using Lemma 5, one can see that relation (17) is positive valued.

Case III: if $n-r \geq 2$, then

$$
\begin{aligned}
{ }^{v} R_{\gamma}(G)-{ }^{v} R_{\gamma}(\dot{G})= & \frac{n-r}{\sqrt{1+\gamma}}\left(\frac{1}{\sqrt{n-r+\gamma+2}}-\frac{1}{\sqrt{n-r+\gamma+3}}\right) \\
& +\frac{2}{\sqrt{2+\gamma}}\left(\frac{1}{\sqrt{n-r+\gamma+2}}-\frac{1}{\sqrt{n-r+\gamma+3}}\right) \\
& +\frac{1}{\sqrt{(2+\gamma)(2+\gamma)}}-\frac{1}{\sqrt{(1+\gamma)(n-r+\gamma+3)}}, \\
{ }^{v} R_{\gamma}(G)-{ }^{v} R_{\gamma}(\dot{G})> & \frac{1}{\sqrt{(2+\gamma)(2+\gamma)}}-\frac{1}{\sqrt{(1+\gamma)(n-r+\gamma+3)}}>0 . \\
& \text { Since } \frac{n-r}{\sqrt{1+\gamma}}\left(\frac{1}{\sqrt{n-r+\gamma+2}}\right. \\
& \left.-\frac{1}{\sqrt{n-r+\gamma+3}}\right)>0 \text { and } \\
& \frac{2}{\sqrt{2+\gamma}}\left(\frac{1}{\sqrt{n-r+\gamma+2}}\right. \\
& \left.-\frac{1}{\sqrt{n-r+\gamma+3}}\right)>0 .
\end{aligned}
$$

2.3. Transformation 3. Let $G \in U_{n}$ with $|G|=n$ and $u, v \in V(G)$ are on the unique cycle of $G$. Pendant neighbors of $u$ and $v$ are $u_{i}$, where $1 \leq i \leq s$, and $v_{j}$, where $1 \leq j \leq t$, respectively. Assume that the path between $u$ and $v$ on cycle is $P_{u v}$ such that $\left|E\left(P_{u v}\right)\right|=r, d\left(V\left(P_{u v}\right)\right)=2$ if $r \geq 2$ and $\left|E\left(P_{v u}\right)\right| \geq 3$. Construct $G$ from $G$ by removing the edges $u u_{i}$ and $v v_{j}$, reducing the path $P_{u v}$ into one vertex $u(v)$ and attaching a star $K_{1, r+s+t+1}$ to $u(v)$, by making sure that $|G|=|G|$. $G$ and $G$ are depicted in Figure 2.

Lemma 8. Let $G, \dot{G} \in U_{n}$ such that $|\dot{G}|=|G|$. By applying Transformation 3 and for $\gamma \geq 0, s, t \geq 1$, we have

$$
{ }^{v} R_{\gamma}(G)>{ }^{v} R_{\gamma}(\dot{G}) \text {. }
$$

Proof

Case I: let $r=1$ and $u v \in E(G)$, then

$$
\begin{aligned}
{ }^{v} R_{\gamma}(G)-{ }^{v} R_{\gamma}(G)> & \frac{s}{\sqrt{(1+\gamma)(s+2+\gamma)}} \\
& +\frac{t}{\sqrt{(1+\gamma)(t+2+\gamma)}} \\
& +\frac{1}{\sqrt{(s+2+\gamma)(t+2+\gamma)}} \\
& -\frac{s+t+1}{\sqrt{(1+\gamma)(s+t+3+\gamma)}}, \\
{ }^{v} R_{\gamma}(G)-{ }^{v} R_{\gamma}(G)> & \frac{s}{\sqrt{1+\gamma}}\left(\frac{1}{\sqrt{s+2+\gamma}}-\frac{1}{\sqrt{s+t+3+\gamma}}\right) \\
& +\frac{t}{\sqrt{1+\gamma}}\left(\frac{1}{\sqrt{t+2+\gamma}}-\frac{1}{\sqrt{s+t+3+\gamma}}\right) \\
& +\frac{1}{\sqrt{(s+2+\gamma)(t+2+\gamma)}} \\
& -\frac{1}{\sqrt{(1+\gamma)(s+t+3+\gamma)}} \\
& \\
& \\
& \\
&
\end{aligned}
$$

Using Lemma 1, one can see that relation (20) is positive valued.

Case II: now let $r \geq 2$, then

$$
\begin{aligned}
{ }^{v} R_{\gamma}(G)-{ }^{v} R_{\gamma}(G)> & \frac{s}{\sqrt{(1+\gamma)(s+2+\gamma)}}+\frac{t}{\sqrt{(1+\gamma)(t+2+\gamma)}} \\
& +\frac{1}{\sqrt{(s+2+\gamma)(2+\gamma)}}+\frac{1}{\sqrt{(2+\gamma)(t+2+\gamma)}} \\
& +\frac{r-2}{\sqrt{(2+\gamma)(2+\gamma)}} \\
& -\frac{s+t+r}{\sqrt{(1+\gamma)(s+t+r+2+\gamma)}} .
\end{aligned}
$$

Since $r \geq 2$ and keeping in mind the fact that 


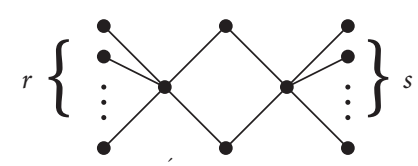

Figure 2: The graph $G$ and $G$ (obtained using Transformation 3).

$$
\Psi(r, s, t)=\frac{s+t+r}{\sqrt{(1+\gamma)(s+t+r+2+\gamma)}},
$$

is an increasing function because

$$
\frac{\partial \Psi}{\partial r}=\frac{s+t+r+4+2 \gamma}{2 \sqrt{\gamma+1}(s+t+r+2+\gamma)^{3 / 2}}>0 .
$$

We have

$$
\begin{aligned}
{ }^{v} R_{\gamma}(G)-{ }^{v} R_{\gamma}(\dot{G})> & \frac{s}{\sqrt{1+\gamma}}\left(\frac{1}{\sqrt{s+2+\gamma}}-\frac{1}{\sqrt{s+t+4+\gamma}}\right) \\
& +\frac{t}{\sqrt{1+\gamma}}\left(\frac{1}{\sqrt{t+2+\gamma}}-\frac{1}{\sqrt{s+t+4+\gamma}}\right) \\
& +\frac{1}{\sqrt{(s+2+\gamma)(2+\gamma)}} \\
& -\frac{1}{\sqrt{(1+\gamma)(s+t+4+\gamma)}} \\
& +\frac{1}{\sqrt{(t+2+\gamma)(2+\gamma)}} \\
& -\frac{1}{\sqrt{(1+\gamma)(s+t+4+\gamma)}}
\end{aligned}
$$

Using Lemma 2, one can see that relation (24) is positive valued.

Let $\mathbf{D}_{n, 3}^{i, j, k}$ be the such class of $\mathbf{D}$ that is obtained from cycle $C_{l}, l=3$ by attaching $K_{1, i}, K_{1, j}$, and $K_{1, k}$ to the vertices of $C_{l}, l=3$. Let $\mathbf{F}_{n, 4}^{r, s}$ be the such class of $\mathbf{F}$ that is obtained from cycle $C_{l}, l=4$ by attaching $K_{1, r}$ and $K_{1, s}$ to the nonadjacent vertices of $C_{l}, l=4$.

Lemma 9. Let $\mathbf{D}_{n, 3}^{i, j, k}, \mathbf{D}_{n, 3}^{i, j-1, k}$, and $\mathbf{D}_{n, 3}^{i+1, j-1, k}$ be three unicyclic graphs in $\mathbf{D}$ as in Figure 3. If $i \geq j \geq 1$, then

$$
{ }^{v} R_{\gamma}\left(\mathbf{D}_{n, 3}^{i, j, k}\right)>{ }^{v} R_{\gamma}\left(\mathbf{D}_{n, 3}^{i+1, j-1, k}\right),
$$

for $\gamma \geq 0$.

Proof. Let $\mathbf{D}_{n, 3}^{i, j, k}, \mathbf{D}_{n, 3}^{i, j-1, k}$, and $\mathbf{D}_{n, 3}^{i+1, j-1, k}$ be three unicyclic graphs in $\mathbf{D}$ as in Figure 3.

$$
\begin{aligned}
{ }^{v} R_{\gamma}\left(\mathbf{D}_{n, 3}^{i, j, k}\right)-{ }^{v} R_{\gamma}\left(\mathbf{D}_{n, 3}^{i+1, j-1, k}\right)= & \frac{j}{\sqrt{(1+\gamma)(j+2+\gamma)}}-\frac{j-1}{\sqrt{(1+\gamma)(j+1+\gamma)}} \\
& +\frac{1}{\sqrt{(i+2+\gamma)(j+2+\gamma)}}-\frac{1}{\sqrt{(i+2+\gamma)(j+1+\gamma)}} \\
& +\frac{1}{\sqrt{(k+2+\gamma)(j+2+\gamma)}}-\frac{1}{\sqrt{(k+2+\gamma)(j+1+\gamma)}}, \\
{ }^{v} R_{\gamma}\left(\mathbf{D}_{n, 3}^{i, j, k}\right)-{ }^{v} R_{\gamma}\left(\mathbf{D}_{n, 3}^{i+1, j-1, k}\right)= & \frac{1}{\sqrt{1+\gamma}}(\sqrt{j+2+\gamma}-\sqrt{j+1+\gamma}) \\
& +\left(\frac{1}{\sqrt{i+2+\gamma}}+\frac{1}{\sqrt{k+2+\gamma}}-\frac{2+\gamma}{\sqrt{1+\gamma}}\right)\left(\frac{1}{\sqrt{j+2+\gamma}}-\frac{1}{\sqrt{j+1+\gamma}}\right), \\
{ }^{v} R_{\gamma}\left(\mathbf{D}_{n, 3}^{i, j, k}\right)-{ }^{v} R_{\gamma}\left(\mathbf{D}_{n, 3}^{i+1, j-1, k}\right)= & \frac{1}{\sqrt{1+\gamma}}\left(\frac{1}{2} \xi_{1}^{\frac{-1}{2}}\right)+\left(\frac{1}{\sqrt{i+2+\gamma}}+\frac{1}{\sqrt{k+2+\gamma}}-\frac{2+\gamma}{\sqrt{1+\gamma}}\right)\left(\frac{-1}{2} \xi_{2}^{\frac{-3}{2}}\right),
\end{aligned}
$$




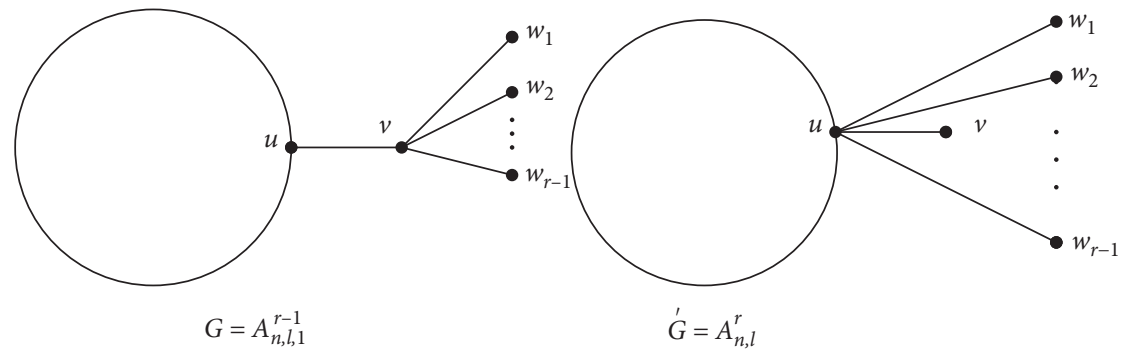

FIgure 3: The graph $\mathbf{D}_{n, 3}^{i, j, k}$ (used in Lemma 9).

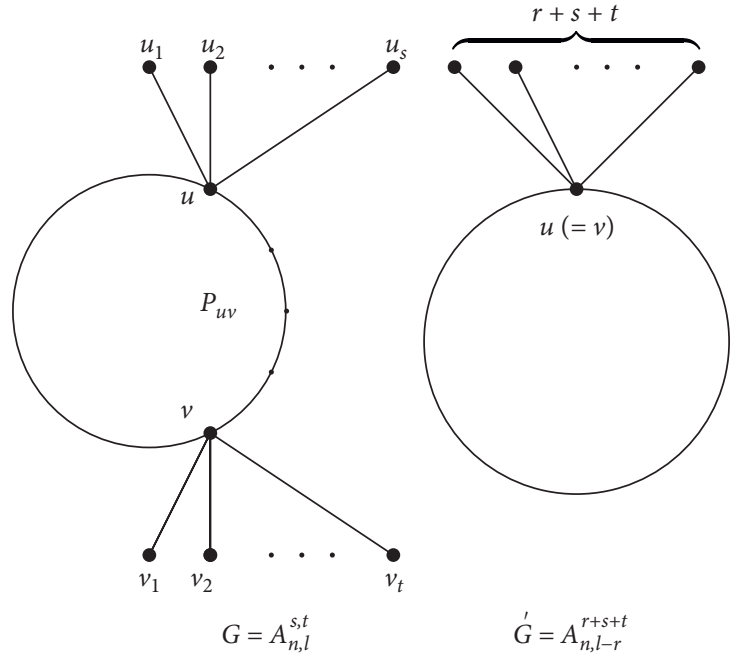

FIgURE 4: The graph $\mathbf{F}_{n, 4}^{r, s}$ (used in Lemma 10).

where $\xi_{1}, \xi_{2} \in(j+1, j+2)$. Similarly, we have

$$
\begin{aligned}
{ }^{v} R_{\gamma}\left(\mathbf{D}_{n, 3}^{i+1, j-1, k}\right)-{ }^{v} R_{\gamma}\left(\mathbf{D}_{n, 3}^{i, j-1, k}\right)= & \frac{i+1}{\sqrt{(1+\gamma)(i+3+\gamma)}}-\frac{i}{\sqrt{(1+\gamma)(i+2+\gamma)}} \\
& +\frac{1}{\sqrt{(i+3+\gamma)(j+1+\gamma)}}-\frac{1}{\sqrt{(i+2+\gamma)(j+1+\gamma)}} \\
& +\frac{1}{\sqrt{(k+2+\gamma)(i+3+\gamma)}}-\frac{1}{\sqrt{(k+2+\gamma)(i+2+\gamma)}}, \\
& +\left(\frac{1}{\sqrt{j+1+\gamma}}+\frac{1}{\sqrt{k+2+\gamma}}-\frac{\gamma+2}{\sqrt{1+\gamma}}\right)\left(\frac{1}{\sqrt{i+3+\gamma}}-\frac{1}{\sqrt{i+2+\gamma}}\right) \\
{ }^{v} R_{\gamma}\left(\mathbf{D}_{n, 3}^{i+1, j-1, k}\right)-{ }^{v} R_{\gamma}\left(\mathbf{D}_{n, 3}^{i, j-1, k}\right)= & \frac{1}{\sqrt{1+\gamma}}(\sqrt{i+3+\gamma}-\sqrt{i+2+\gamma}) \\
{ }^{v} R_{\gamma}\left(\mathbf{D}_{n, 3}^{i+1, j-1, k}\right)-{ }^{v} R_{\gamma}\left(\mathbf{D}_{n, 3}^{i, j-1, k}\right)= & \frac{1}{\sqrt{1+\gamma}}\left(\frac{1}{2} \xi^{-1 / 2}\right)+\left(\frac{1}{\sqrt{j+1+\gamma}}+\frac{1}{\sqrt{k+2+\gamma}}-\frac{\gamma+2}{\sqrt{1+\gamma}}\right)\left(\frac{-1}{2} \xi_{3}^{-1 / 2}\right), \\
\text { where } \xi_{3}, \xi_{4} \in(i+2, i+3) . & { }^{v} R_{\gamma}\left(D_{n, 3}^{i, j, k}\right)-{ }^{v} R_{\gamma}\left(D_{n, 3}^{i, j-1, k}\right)>{ }^{v} R_{\gamma}\left(D_{n, 3}^{i+1, j-1, k}\right)-{ }^{v} R_{\gamma}\left(D_{n, 3}^{i, j-1, k}\right) .
\end{aligned}
$$

Bearing in mind the fact that $i \geq j$, one can see that 
Hence, ${ }^{v} R_{\gamma}\left(\mathbf{D}_{n, 3}^{i, j, k}\right)>{ }^{v} R_{\gamma}\left(\mathbf{D}_{n, 3}^{i+1, j-1, k}\right)$.

Lemma 10. Let $\mathbf{F}_{n, 4}^{r, s}, \mathbf{F}_{n, 4}^{r, s-1}$, and $\mathbf{F}_{n, 4}^{r+1, s-1}$ be three unicyclic graphs in $\mathbf{F}$ as in Figure 4. If $r \geq s \geq 1$, then

$$
{ }^{v} R_{\gamma}\left(\mathbf{F}_{n, 4}^{r, s}\right)>{ }^{v} R_{\gamma}\left(\mathbf{F}_{n, 4}^{r+1, s-1}\right),
$$

for $\gamma \geq 0$.

Proof. Let $\Psi(t)=(t / \sqrt{1+\gamma}+2 / \sqrt{2+\gamma})(1 / \sqrt{t+2+\gamma})$ and keeping in mind Figure 4 , we have

$$
\begin{aligned}
& { }^{v} R_{\gamma}\left(F_{n, 4}^{r, s}\right)=\frac{r}{\sqrt{(1+\gamma)(r+2+\gamma)}} \\
& +\frac{s}{\sqrt{(1+\gamma)(s+2+\gamma)}} \\
& +\frac{2}{\sqrt{(2+\gamma)(r+2+\gamma)}} \\
& +\frac{2}{\sqrt{(2+\gamma)(s+2+\gamma)}} \\
& { }^{v} R_{\gamma}\left(F_{n, 4}^{r, s-1}\right)=\frac{r}{\sqrt{(1+\gamma)(r+2+\gamma)}} \\
& +\frac{s-1}{\sqrt{(1+\gamma)(s+1+\gamma)}} \\
& +\frac{2}{\sqrt{(2+\gamma)(r+2+\gamma)}} \\
& +\frac{2}{\sqrt{(2+\gamma)(s+1+\gamma)}} \\
& { }^{v} R_{\gamma}\left(F_{n, 4}^{r+1, s-1}\right)=\frac{r+1}{\sqrt{(1+\gamma)(r+3+\gamma)}} \\
& +\frac{s-1}{\sqrt{(1+\gamma)(s+1+\gamma)}} \\
& +\frac{2}{\sqrt{(2+\gamma)(r+3+\gamma)}} \\
& +\frac{2}{\sqrt{(2+\gamma)(s+1+\gamma)}} \\
& { }^{v} R_{\gamma}\left(F_{n, 4}^{r, s}\right)-{ }^{v} R_{\gamma}\left(F_{n, 4}^{r, s-1}\right)=\left(\frac{s}{\sqrt{1+\gamma}}+\frac{2}{\sqrt{2+\gamma}}\right) \frac{1}{\sqrt{s+2+\gamma}} \\
& -\left(\frac{s-1}{\sqrt{1+\gamma}}+\frac{2}{\sqrt{2+\gamma}}\right) \frac{1}{\sqrt{s+1+\gamma}} \\
& { }^{v} R_{\gamma}\left(F_{n, 4}^{r, s}\right)-{ }^{v} R_{\gamma}\left(F_{n, 4}^{r, s-1}\right)=\Psi(s)-\Psi(s-1)=\Psi^{\prime}\left(\xi_{1}\right),
\end{aligned}
$$

where $\xi_{1} \in(s+1, s+2)$. Similarly, we have

$$
\begin{aligned}
{ }^{v} R_{\gamma}\left(\mathbf{F}_{n, 4}^{r+1, s-1}\right)-{ }^{v} R_{\gamma}\left(\mathbf{F}_{n, 4}^{r, s-1}\right)= & \left(\frac{r+1}{\sqrt{1+\gamma}}+\frac{2}{\sqrt{2+\gamma}}\right) \\
& \cdot \frac{1}{\sqrt{r+3+\gamma}} \\
& -\left(\frac{a}{\sqrt{1+\gamma}}+\frac{2}{\sqrt{2+\gamma}}\right) \\
& \cdot \frac{1}{\sqrt{r+2+\gamma}}, \\
{ }^{v} R_{\gamma}\left(\mathbf{F}_{n, 4}^{r+1, s-1}\right)-{ }^{v} R_{\gamma}\left(\mathbf{F}_{n, 4}^{r, s-1}\right)= & \Psi(r+1)-\Psi(r)=\Psi \prime\left(\xi_{2}\right),
\end{aligned}
$$

where $\xi_{2} \in(r+2, r+3)$.

Bearing in mind the fact that $r \geq s \geq 1, \quad \Psi^{\prime \prime}(t)=$ $-1 / 2(t+2+\gamma)^{5 / 2}(2(t+2+\gamma) / \sqrt{1+\gamma}-(3 / 2)\{t / \sqrt{1+\gamma}+2 /$ $\sqrt{2+\gamma}\})<0$ for all $t>0$. Also, $\Psi^{\prime}\left(\xi_{1}\right)-\Psi^{\prime}\left(\xi_{2}\right)>0$, which implies that ${ }^{v} R_{\gamma}\left(\mathbf{F}_{n, 4}^{r, s}\right)>{ }^{v} R_{\gamma}\left(\mathbf{F}_{n, 4}^{r+1, s-1}\right)$.

As a consequence of Lemmas 6-10, one has the following.

Theorem 1. Among all the unicyclic graphs of order $n \geq 4, S_{n}^{1}$ has the minimum variable Randic index for $\gamma \geq 0$ and its value is

$$
\begin{aligned}
{ }^{v} R_{\gamma}(G) \geq & \frac{n-3}{\sqrt{(1+\gamma)(n-1+\gamma)}}+\frac{2}{\sqrt{(2+\gamma)(n-1+\gamma)}} \\
& +\frac{1}{\sqrt{(2+\gamma)(2+\gamma)}} .
\end{aligned}
$$

\section{Data Availability}

No data were used to support this study.

\section{Conflicts of Interest}

The authors declare that they have no conflicts of interest.

\section{Acknowledgments}

This work was supported by the National University of Computer and Emerging Sciences, Lahore, Pakistan.

\section{References}

[1] J. L. Gross, J. Yellen, Graph Theory, CRC Press, Boca Raton, FL, USA, 2000

[2] F. Harary, Graph Theory, Addison-Wesley, Boston, MA, USA, 1969.

[3] N. Trinajstić, Chemical Graph Theory, CRC Press, Boca Raton, FL, USA, 1992

[4] M. Randić, M. Pompe, D. Mills, and S. C. Basak, "Variable connectivity index as a tool for modeling structure-property relationships," Molecules (Basel, Switzerland), vol. 9, no. 12, pp. 1177-1193, 2004. 
[5] M. Randić, "On computation of optimal parameters for multivariate analysis of structure-property relationship," Journal of Computational Chemistry, vol. 12, no. 8, pp. 970980, 1992.

[6] M. Randić, "Novel graph theoretical approach to heteroatoms in quantitative structure-activity relationships," Chemometrics and Intelligent Laboratory Systems, vol. 10, pp. 213227, 1991.

[7] M. Randić, "On history of the Randić index and emerging hostility toward chemical graph theory," MATCH Communications in Mathematical and in Computer Chemistry, vol. 59, pp. 5-124, 2008.

[8] M. Randić, "On characterization of molecular branching," Journal of the American Chemical Society, vol. 97, no. 23, pp. 6609-6615, 1975.

[9] J. C. Dearden, "The use of topological indices in QSAR and QSPR modeling," in Advances in QSAR Modeling, K. Roy, Ed., pp. 57-88, Springer, Cham, Switzerland, 2017.

[10] R. G. Domenech, J. Gálvez, J. V. de Julián-Ortiz, and L. Pogliani, "Some new trends in chemical graph theory," Chemical Reviews, vol. 108, no. 3, pp. 1127-1169, 2008.

[11] D. Liu and C. Zhong, "Modeling of the heat capacity of polymers with the variable connectivity index," Polymer Journal, vol. 34, no. 12, pp. 954-961, 2002.

[12] M. Pompe, "Variable connectivity index as a tool for solving the anti connectivity problem," Chemical Physics Letters, vol. 404, pp. 296-299, 2005.

[13] M. Randic and S. C. Bašak, "On use of the variable connectivity index ${ }^{1} \chi^{f}$ in QSAR: toxicity of aliphatic ethers," Journal of Chemical Information and Computer Sciences, vol. 41, no. 3, pp. 614-618, 2001.

[14] M. Randic, "Characterization of molecular branching," Journal of the American Chemical Society, vol. 97, no. 23, pp. 6609-6615, 1975.

[15] M. Randić, D. Plavšić, and N. Leřs, "Variable connectivity index for cycle-containing structures," Journal of Chemical Information and Computer Sciences, vol. 41, no. 3, pp. 657662, 2001.

[16] M. Randić, "On the history of the connectivity index: from the connectivity index to the exact solution of the protein alignment problem," SAR and QSAR in Environmental Research, vol. 26, no. 7-9, pp. 523-555, 2015.

[17] M. Randić and M. Pompe, "The variable connectivity index ${ }^{1} \chi^{f}$ versus the traditional molecular descriptors: a comparative study of ${ }^{1} \chi^{f}$ against descriptors of codessa," Journal of Chemical Information and Computer Sciences, vol. 41, no. 3, pp. 631-638, 2001.

[18] S. Yousaf, A. A. Bhatti, and A. Ali, "Minimum variable connectivity index of trees of a fixed order," Discrete Dynamics in Nature and Society, In press.

[19] A. Ali, Z. Du, and K. Shehzadi, "Estimating some general molecular descriptors of saturated hydrocarbons," Molecular Informatics, vol. 38, Article ID 1900007, 2019.

[20] D. Amić, D. Davidovic-Amić, D. Bešlo, B. Lučić, N. Trinajstić, and S. Nikolić, "The vertex-connectivity index revisited," Journal of Chemical Information and Computer Sciences, vol. 38, no. 5, pp. 819-822, 1998.

[21] A. Behtoei, "Some relations and bounds for the general first zagreb index," MATCH Communications in Mathematical and in Computer Chemistry, vol. 81, pp. 361-370, 2019.

[22] D. Dimitrov and A. Ali, "On the extremal graphs with respect to the variable sum exdeg index," Discrete Mathematics Letters, vol. 1, pp. 42-48, 2019.
[23] S. Elumalai and T. Mansour, "On the general zeroth-order Randić index of bargraphs," Discrete Mathematics Letters, vol. 2, pp. 6-9, 2019.

[24] F. Hayat, "On generalized atom-bond connectivity index of cacti," Iranian Journal of Mathematical Chemistry, vol. 10, pp. 319-330, 2019.

[25] A. Martínez-Pérez and J. M. Rodríguez, "A unified approach to bounds for topological indices on trees and applications, MATCH Commun," Mathematical and in Computer Chemistry, vol. 82, pp. 679-698, 2019. 